Nervenarzt 2006 $\cdot 77: 1064-1070$ DOI 10.1007/s00115-005-2044-0

Online publiziert: 20. Januar 2006

๑) Springer Medizin Verlag 2006

G. Wiesbeck · K. Dürsteler-MacFarland

Bereich Abhängigkeitserkrankungen, Universitäre Psychiatrische Kliniken, Basel

\title{
Neue Entwicklungen in der Pharmakotherapie der Kokainabhängigkeit
}

kompliziert sind und auch andere Neurotransmittersysteme, insbesondere das serotonerge, zur Vermittlung suchterzeugender Kokaineffekte beitragen [24, 52].

Eine wirksame Pharmakotherapie zur Reduktion des Rückfallrisikos bzw. zur Verringerung der Konsummenge existierte für Kokainabhängige lange Zeit nicht. In einer systematischen Übersichtarbeit referierten Lima et al. [38] den aktuellen Stand der Pharmakotherapie bis zum Jahr 200o. Sie sichteten dafür über 1000 Veröffentlichungen, unter denen sie 49 randomisierte, kontrollierte Studien nach methodischen Gütekriterien auswählten und analysierten. Eine Aussage war damals zu folgenden Substanzen möglich: Bupropion, Desipramin, Fluoxetin, Gepiron, Imipramin, Ritanserin, Amantadin, Bromocriptin, Perolid, Carbamazepin, Mazindol, Naltrexon, Phenytoin und Risperidon. Für keine dieser Substanzen fanden die Autoren einen überzeugenden Wirksamkeitsnachweis. Sie kamen daher zu dem Schluss, dass es bis zum Jahre 2000 keine Evidenz für eine wirksame Pharmakotherapie der Kokainabhängigkeit gäbe [38].

Mittlerweile sind jedoch neue Kandidatensubstanzen aufgetaucht, von denen die vielversprechendsten hier vorgestellt und bewertet werden sollen. Dabei beschränkt sich diese Übersicht auf jene, die innerhalb der letzten fünf Jahre am Menschen getestet wurden oder derzeit noch erprobt werden.

\section{Potenzielle \\ Kandidatensubstanzen}

Vigabatrin

Nach Aussage des National Institute on Drug Abuse (NIDA) nähert sich Vigabatrin erfolgreich dem Stadium der klinischen Zulassungsprüfungen (http:// www.drugabuse.gov/NIDA_notes/NNVol18N6/Long.html). Das als Antiepileptikum auch in Europa zugelassene Medikament hemmt selektiv und irreversibel die GABA-Transaminase, welche für den Abbau von GABA ( $\gamma$-Aminobuttersäure) verantwortlich ist und bewirkt dadurch eine Erhöhung der GABA-Konzentration im Gehirn.

Nachdem die Substanz im Tierversuch eine kokaininduzierte Dopaminfreisetzung im Nucleus accumbens zu hemmen vermochte [54], wurde Vigabatrin nun erstmals auch am Menschen erfolgreich erprobt: Von 20 kokainabhängigen Studienteilnehmern, die mit Vigabatrin offen behandelt wurden, beendeten 8 die Untersuchung und zeigten dabei kokainkonsumfreie Zeiträume zwischen 46 und 58 Tagen. Sechs dieser Studienbeender blieben sogar vollkommen kokainabstinent. Fast alle berichteten von einem Verlust des Kokaincravings zwischen der zweiten und dritten Behandlungswoche [5]. Dies macht Vigabatrin zu einer viel versprechenden Substanz, die jedoch wegen der zu geringen Datenlage derzeit noch nicht zur Behandlung empfohlen werden kann. 
Hier steht eine Anzeige.

黛 Springer 


\section{Tiagabin}

Dieses als Antiepileptikum zur Addon-Behandlung therapieresistenter fokaler Anfälle zugelassene Medikament verstärkt die GABAerge Wirkung durch Hemmung der GABA-Wiederaufnahme. In einer ersten randomisierten Pilotuntersuchung wurden 45 Kokainabhängige aus einem Methadonsubstitutionsprogramm mit zwei Dosierungen Tiagabin (12 mg/Tag bzw. 24 mg/Tag) oder mit Plazebo behandelt. Zielvariable war die Veränderung kokainfreier Urinproben innerhalb einer Behandlungsgruppe nach 10-wöchiger Behandlung (vorher/nachher Vergleich). In der Hochdosisgruppe (24 mg/Tag) stieg die Anzahl kokainfreier Urinproben signifikant um $33 \%$ an, in der Gruppe mit 12 mg/Tag nur um 14\%. Dagegen nahm unter Plazebobehandlung die Anzahl kokainfreier Urinproben um 10\% ab [22]. Dieses Ergebnis empfiehlt Tiagabin für weitere klinische Prüfungen.

\section{Selegilin}

Selegilin, ein spezifischer Monoamin-Oxidase-B- (MAO-B-)Inhibitor zeigte in $\mathrm{Hu}$ manversuchen mit kleiner Fallzahl und experimentellem Design eine Reduktion der Kokaineffekte, insbesondere des subjektiven „High-Erlebens“ $[2,29,46]$. Selegilin könnte daher ebenfalls eine Kandidatensubstanz für weitergehende klinische Wirksamkeitsuntersuchungen sein.

\section{Baclofen}

Der selektive GABA-B-Rezeptor-Agonist Baclofen, der im Tierversuch u. a. die durch Kokain induzierte Dopaminfreisetzung im Nucleus accumbens antagonisierte [15], wurde mittlerweile in drei Untersuchungen auch am Menschen erprobt. In einer ersten Pilotstudie wurden 1o Kokainkonsumenten 16 Wochen lang mit $60 \mathrm{mg}$ Baclofen/Tag offen behandelt [39]. Die Patienten berichteten von einer Reduktion sowohl des Kokaincravings als auch des Kokainkonsums. Dagegen fanden Lile et al. [37] in einer experimentellen Untersuchung bei 7 Kokainkonsumenten keinen Einfluss von Baclofen auf das Konsumverhalten. In einer kontrollierten klinischen Studie mit 70 Kokainab- hängigen zeigte die Substanz keine Überlegenheit gegenüber Plazebo [56]. Baclofen kann daher nicht für die Behandlung Kokainabhängiger empfohlen werden.

\section{Topiramat}

In einer kontrollierten klinischen Pilotstudie mit kleiner Fallzahl ( $n=40$ Kokainabhängige) wurde Topiramat 13 Wochen lang bis zu einer Höhe von $200 \mathrm{mg} /$ Tag gegeben. Im Vergleich zu Plazebo erhöhte Topiramat nicht nur die Abstinenzwahrscheinlichkeit, es blieben unter Topiramat auch signifikant mehr Kokainabhängige durchgehend abstinent [31]. Topiramat hat sich damit als eine Kandidatensubstanz für weitergehende klinische Prüfungen bei Kokainabhängigkeit empfohlen [48].

\section{Modafinil}

Modafinil ist ein Psychoanaleptikum zur Behandlung von Narkolepsien. In einer kontrollierten Interaktionsstudie mit experimentellem Design $(n=7)$ wurde die Wirkung von niedrig- (200 mg/Tag) und hochdosiertem (40o mg/Tag) Modafinil auf das subjektives Erleben nach Kokainapplikation (30 mg per infusionem) untersucht. Modafinil reduzierte signifikant die durch Kokain ausgelöste Euphorie [11].

Daraufhin wurde von der gleichen Arbeitsgruppe die Substanz in einem ersten klinischen Versuch erprobt. Unter kontrollierten Bedingungen wurden 62 Kokainabhängige 8 Wochen lang doppelblind mit 400 mg/Tag Modafinil oder Plazebo behandelt. In Bezug auf die primäre Zielvariable (kokainfreie Urinproben) fiel der Vergleich statistisch signifikant zugunsten des Modafinil aus [12]. Das National Institute on Drug Abuse (NIDA) hat Modafinil daher als eine viel versprechende Kandidatensubstanz zur Behandlung der Kokainabhängigkeit eingestuft, bei der weitergehende klinische Prüfungen gerechtfertigt sind.

\section{Disulfiram}

Disulfiram, ein jahrzehntealtes Traditionsmedikament zur „Vergällung“ der Alkoholwirkung, das durch die modernen Anticravingsubstanzen zunehmend aus der Alkoholismusbehandlung verdrängt wurde, taucht nun mit neuer Evidenz in der Therapieforschung zur Kokainabhängigkeit wieder auf. Die Substanz hemmt die Aldehyddehydrogenase, was nach dem Konsum von Alkohol zu einem Anstieg der Acetaldehydkonzentration und konsekutiv zur disulfiramtypischen Alkoholunverträglichkeitsreaktion führt (Acetaldehydsnydrom: Übelkeit, Erbrechen, Hautrötung, Herzklopfen, Blutdruckabfall).

Die Exzesskomorbidität von Kokainund Alkoholabhängigkeit war der Ausgangspunkt für Überlegungen, Disulfiram könnte über eine Reduktion des Alkoholkonsums auch zu einer Verringerung der Kokainmenge führen. Diese Komorbidität beruht auf mehreren, sich in idealtypischer Weise ergänzenden Wirkeffekten beider Substanzen. Alkohol verringert durch seine sedierende Wirkung die negativen Folgen des Kokains (z. B. Schlaflosigkeit) bzw. des akuten Kokainentzugs. Über seine enthemmende Wirkung beeinträchtigt er das Urteilsvermögen und damit die Kontrolle über den Kokaingebrauch. Schließlich werden durch das Cocaethylen - ein aktiver Kokainmetabolit, der beim Abbau von Kokain in Anwesenheit von Alkohol entsteht - das euphorische Rauschstadium verlängert, die Symptome des Entzugs gemildert, aber auch die Toxizität des Kokains erhöht [42]. Cocaethylen erwies sich als äquipotent zu Kokain in Bezug auf die Hemmung der synaptischen Rückaufnahme und in der Hemmung des Dopamintransporters [26].

Untersuchungen mit experimentellem Design zeigten, dass eine Vorbehandlung mit Disulfiram nicht nur die kardiovaskulären Effekte intranasal zugeführten Kokains verstärkt, sondern auch dessen aversive Folgen wie Angst und Dysphorie [25, 41, 43]. Dies könnte auf einer Hemmung von kokainmetabolisierenden Serumesterasen durch Disulfiram beruhen [28]. Andererseits hemmt Disulfiram die Dopamin- $\beta$-Hydroxylase $[6,20]$. Die Dopaminwirkung nach Kokainkonsum könnte dadurch mit einer vermehrten Angst- und Dysphoriereaktion verbunden sein. $\mathrm{Zu}-$ sammen führten all diese Befunde zu der begründeten Hypothese, dass Disulfiram den Kokainkonsum einschränkt.

Fallberichte [27] sowie zwei kontrollierte klinische Studien $[7,9]$ erhärteten diese Hypothese. Unter anderem zeigte 
sich dabei ein signifikanter Behandlungseffekt von Disulfiram plus Psychotherapie. Patienten, die auf diese Weise kombiniert behandelt wurden, reduzierten nicht nur ihre Konsummengen an Alkohol und Kokain, sondern verlängerten auch signifikant ihre Abstinenzdauer [9].

Erste Hinweise darauf, dass die Wirksamkeit von Disulfiram nicht an die Komorbidität mit Alkoholismus gebunden ist, ergaben sich in einer kontrollierten Untersuchung mit Disulfiram bei substituierten Opiatabhängigen. Die Diagnose „Alkoholabhängigkeit" war hier explizit als Ausschlusskriterium definiert worden. Kokainabhängige unter Disulfiram wurden im Verlauf der 12-wöchigen Behandlung signifikant häufiger kokainnegativ getestet als Studienteilnehmer unter Plazebo [19].

In einer jüngsten kontrollierten Studie wurde mit einem $2 \times 2$-faktoriellen Design die Wirkung von zwei Formen von Psychotherapie (kognitive Verhaltenstherapie [CBT], interpersonelle Psychotherapie [IPT]) plus zwei Formen der Medikation (Disulfiram, Plazebo) bei 121 Kokainabhängigen getestet. Nach 12-wöchiger Behandlung zeigte sich eine signifikante Überlegenheit von Disulfiram gegenüber Plazebo und von CBT gegenüber IPT. Die größten Behandlungseffekte wurden bei Kokainabhängigen ohne komorbide $\mathrm{Al}$ koholabhängigkeit erzielt [8]. Dies führte zu der Vermutung, dass Disulfiram seine Wirkung unmittelbar auf den Kokainkonsum ausübt und nicht sekundär über eine Veränderung des Begleitalkoholismus.

Unter allen Medikamenten, welche für die Indikation „Kokainabhängigkeit“ bisher getestet wurden, ergibt sich für Disulfiram die größte Evidenz für Behandlungswirksamkeit. Jüngste Ergebnisse deuten jedoch darauf hin, dass eventuell nur Männer von einer Behandlung mit Disulfiram profitieren [47]. Dies spräche für einen geschlechterdifferenten Wirkeffekt, der weiter erforscht werden müsste.

\section{Methylphenidat}

Das strukturchemisch mit dem Amphetamin verwandte und wie dieses dem $\mathrm{Be}$ täubungsmittelgesetz unterliegende $\mathrm{Me}$ thylphenidat entfaltet seine stimulierende, indirekt sympathomimetische Wirkung über die präsynaptische Rückaufnahme- hemmung von Dopamin und vermutlich auch von Noradrenalin [34, 6o]. Methylphenidat zeigt eine dem Kokain vergleichbare Affinität zum Dopamintransporter [50]. PET-Untersuchungen zur Pharmakokinetik mittels radioaktiv $\left({ }^{11} \mathrm{C}\right)$ markiertem Methylphenidat bzw. Kokain zeigten Gemeinsamkeiten, aber auch deutliche Unterschiede [58]. Gemeinsam ist beiden Substanzen die rasche Aufnahme im Gehirn und das regionale Verteilungsmuster mit den höchsten Konzentrationen im Striatum und den niedrigsten in Kortex und Zerebellum. Das subjektive „HighErleben“ trat für beide Substanzen wenige Minuten nach Injektion auf und verschwand vollständig nach $30-40 \mathrm{~min}$. Interessanterweise verlief dazu parallel nur die Pharmakokinetik des Kokains. Methylphenidat hingegen verweilte etwa 4mal länger im Striatum als Kokain, ohne dass sich dies im subjektiven „High-Erleben“ der Probanden widerspiegelte [58, 59]. Es könnte daher sein, dass bei gleicher Anflutungsgeschwindigkeit und vergleichbarem Rauscherleben diese wesentlich langsamere „brain clearance“ für die geringere Selbstapplikationsfrequenz und damit für das geringere Missbrauchsrisiko des Methylphenidats im Vergleich zu Kokain mit verantwortlich ist [49]. Darüber hinaus sprechen die Befunde dafür, dass die Blockade striataler Dopamintransporter mit Methylphenidat nicht ausreicht, um ein dem Kokain vergleichbares „High-Gefühl“ zu induzieren [59]. Dies wurde mit einer experimentellen Untersuchung bestätigt, bei der die Wirkung von Methylphenidat bei Kokainabhängigen untersucht wurde. Es zeigte sich, dass die positiven Wirkungen der Methylphenidat nicht ausreichten, um die Kokainwirkung adäquat ersetzen zu können [51].

In einer klinischen Untersuchung an 49 Kokainabhängigen mit einer Aufmerksamkeits-Defizits-Hyperaktivitäts-Störung (ADHS), die 10 Wochen lang offen mit Methylphenidat (6o mg/Tag p.o.) behandelt wurden, zeigte sich eine durchgehende Kokainabstinenz bei 37\% der komplianten, dagegen bei o\% der nonkomplianten Studienteilnehmer [57].

In der bislang einzigen plazebokontrollierten klinischen Studie wurde Methylphenidat (45 mg/Tag p.o.) 11 Wochen lang als Substitut bei Kokainabhängigen ohne
Nervenarzt $2006 \cdot 77: 1064-1070$

DOI 10.1007/s00115-005-2044-0

(c) Springer Medizin Verlag 2006

\section{G. Wiesbeck $\cdot$ K. Dürsteler-MacFarland Neue Entwicklungen in der Pharmakotherapie der Kokainabhängigkeit}

\section{Zusammenfassung}

Es zeichnen sich neue medikamentöse Behandlungsmöglichkeiten der Kokainabhängigkeit ab. So gelang es erstmals mit Disulfiram in einer randomisierten, kontrollierten Studie, den Kokainkonsum signifikant zu reduzieren. Im Oktober 2003 startete in den USA eine Phase-Ilb-Studie mit aktiver Immunisierung gegen Kokain, seit September 2004 läuft in der Schweiz eine Behandlungsstudie mit Methylphenidat in Kombination mit kognitiver Verhaltenstherapie. Vigabatrin, Tiagabin, Selegilin und Topiramat haben sich in Pilotuntersuchungen als potenzielle Kandidatensubstanzen für die weitere klinische Prüfung empfohlen.

Schlüsselwörter

Kokainabhängigkeit · Disulfiram · Aktive Immunisierung $\cdot$ Methylphenidat - Vigabatrin . Tiagabin · Selegilin · Topiramat

\section{New developments in the pharmacotherapy of cocaine dependence}

\section{Summary}

New possibilities with medications for the treatment of cocaine dependence have begun to emerge. For example, in a randomised controlled study, disulfiram succeeded for the first time in significantly reducing cocaine consumption. In October 2003, a phase llb study was started in the USA testing active immunisation against cocaine dependence. There is also an ongoing study in Switzerland testing methylphenidate treatment in combination with cognitive behavioural therapy. Pilot studies indicate that vigabatrin, selegiline, and topiramate are promising candidates for further clinical substance testing.

\section{Keywords}

Cocaine dependence - Disulfiram · Active immunisation · Methylphenidate $\cdot$ Vigabatrin Selegiline - Topiramate 
ADHS erprobt. In Bezug auf Haltequote und Kokainkonsum konnte keine Überlegenheit von Methylphenidat gegenüber Plazebo nachgewiesen werden [21]. Allerdings litt die Studie unter einer zu geringen Fallzahl $(n=24)$, so dass kleine oder mittlere Wirkeffekte damit nicht nachgewiesen werden konnten.

Die Vermutung, dass eine Behandlung mit Stimulanzien, wie z. B. Methylphenidat, den Kokainkonsum steigern könnte („priming effect“), konnte nicht bestätigt werden [18].

\section{Schweizerische Behandlungsstudie mit Methylphenidat}

Ein besonderes Problem stellt der Kokainkonsum bei Opiatabhängigen dar, der häufig exzessiv („binge use“) und intravenös erfolgt $[16,36]$. In einer klinischen Behandlungsstudie wird bei dieser Gruppe von Patienten derzeit doppelblind und plazebokontrolliert die Einsatzmöglichkeit von Methylphenidat mit und ohne kognitiv-behaviorale Psychotherapie (CBT) untersucht. Primäre Zielvariablen sind die Studienverweildauer, das Kokainverlangen sowie Häufigkeit und Menge des Kokainkonsums. Die Rekrutierungsphase ist abgeschlossen, die Ergebnisse werden 2006 vorliegen.

\section{Impfung}

Erste Versuche, das Prinzip der Immunisierung für die Suchttherapie zu nutzen, gehen in die 197oer Jahre zurück. Im Tierexperiment war es damals gelungen, einen zur freiwilligen Selbstapplikation von $\mathrm{He}$ roin und Kokain trainierten Rhesusaffen spezifisch gegen Heroin zu immunisieren und damit das Selbstapplikationsverhalten des Tieres selektiv für Heroin zu löschen [4].

Zur Anwendung im Humanversuch gelangte die aktive Immunisierung jedoch erst mit der Entwicklung eines Kokain-Protein-Konjugats, das eine Immunreaktion mit ausreichender Antikörperproduktion auslösen konnte [17] und bei immunisierten Ratten die freiwillige Selbstapplikation von Kokain signifikant reduzierte [32]. Es handelt sich dabei um ein Proteinkonjugat, in dem Succinylnorcocain (SNC) an rekombinantes Choleratoxin $\mathrm{B}$ (rCTB) gekoppelt wurde, das sich beim Menschen als sicheres Trägerprotein etabliert hat. Dieser Impfstoff ist unter der Bezeichnung TA-CD (Xenova Group plc, Berkshire, UK) als Antikokain-Vakzine mittlerweile in einer Phase-I-Prüfung mit drei Dosierungen an 34 ehemaligen Kokainkonsumenten auf Sicherheit und Immunogenität erfolgreich geprüft worden [33]. Die ersten Anti-Kokain-Antikörper konnten am Tag 28 (14 Tage nach der 2. Impfung) nachgewiesen werden, wobei die Höhe der Antikörperantwort mit der Impfdosis korrelierte. Antikörpertiter, die nach der 3. Impfung ihr Maximum erreichten, blieben über 4 Monate stabil und fielen nach einem Jahr wieder auf Ausgangsniveau zurück [33].

Informationen über zwei aktuell laufende Phase-II-Studien sind momentan nur über Presseverlautbarungen im Internet erhältlich (http://www.xenova.co.uk/ PressReleases). Demnach begann die erste Phase-IIa-Studie im April 2002. Untersucht wurde die Sicherheit und Immunogenität von TA-CD mit einem Impfplan von 4 bzw. 5 Dosierungen. Wie bereits in der Phase-I-Studie erwies sich die Impfung als sicher und die Antikörperreaktion als dosiskorreliert. Von 16 Patienten, die während der Studie Kokain konsumierten, berichteten 14 von einer Reduktion der euphorischen Rauschwirkung.

Eine zweite Phase-IIa-Studie begann im April 2003 in den USA. Zehn Patienten wurden hier offen behandelt. Untersucht wurden die Auswirkungen einer Impfung mit TA-CD auf Verhaltensänderungen nach Kokainkonsum.

Im Oktober 2003 wurde der Beginn der ersten Phase-IIb-Studie angekündigt. Sie wird zurzeit an der Yale Universität in den USA an 132 methadonsubstituierten Kokainabhängigen durchgeführt. Randomisiert und doppelblind erhalten die Teilnehmer entweder aktives TA-CD oder Plazebo. Sie werden 3-mal pro Woche über einen Zeitraum von 20 Wochen nach ihrem Kokainkonsum befragt (einschließlich Urintestung auf Kokainmetabolite). Die Ergebnisse werden für 2006 erwartet (http:// www.xenova.co.uk/PressReleases).

\section{Katalytische Antikörper}

Auf dem Prinzip der passiven Immunisierung beruhend werden monoklonale An- tikörper appliziert, die an Kokain binden, es hydrolysieren und danach für weitere katalytische Zyklen zur Verfügung stehen. Dadurch, so die Hypothese, könnte die Serumkokainkonzentration in einem Maß gesenkt werden, dass ein ReinforcementEffekt nicht mehr auftreten kann [35, 44]. Der bisher nur im Tierversuch angewendete katalytische monoklonale Antikörper 15A1o zeigte allerdings keine ausreichende Aktivität [61]. Wurden hohe Dosen an Kokain intravenös zugeführt, reichte der Impfschutz lediglich für 72 h [1]. Die Entwicklung polyklonaler Antikörper mit höherer katalytischer Aktivität könnte zukünftig einen verlängerten Impfschutz ermöglichen $[3,10]$. Im Gegensatz zur aktiven Immunisierung ist bei den katalytischen Antikörpern eine klinische Erprobung derzeit allerdings noch nicht in Sicht.

\section{Fazit für die Praxis}

Die medikamentöse Behandlung der Kokainabhängigkeit basierte jahrzehntelang auf Einzelfallschilderungen und klinischen Untersuchungen mit unzulänglicher Methodik. Empfehlungen entsprangen eher einem therapeutischen Wunschdenken als tatsächlichen Wirksamkeitsnachweisen. So gibt es beispielsweise bis heute keine ausreichende Evidenz für den Gebrauch von Carbamazepin, Amantadin, Bromocriptin oder Lithium, und insbesondere die zahlreichen tradierten Empfehlungen für die Anwendung von Desipramin und Imipramin genügen nicht mehr den heutigen Anforderungen eines wissenschaftlich fundierten Wirksamkeitsnachweises.

Allein Disulfiram, das von seinem Wirkmechanismus her nicht in den engeren Kreis potenzieller Kandidatensubstanzen gehörte, konnte bislang seine Wirksamkeit in einer methodisch anspruchvollen Studie unter Beweis stellen. Dies entspräche nach der Diktion moderner Behandlungsleitlinien immerhin der Empfehlungsklasse lb, $d$. $h$. Evidenz aufgrund mindestens einer randomisierten, kontrollierten Studie [23]. Könnte dieses Ergebnis reproduziert werden, so stände neben den nachgewiesenermaßen wirksamen Medikamenten bei Alkoholismus (Acamprosat, Naltrexon) und Tabakabhängigkeit (Bupropion) mit Disulfiram bei Kokainabhän- 
gigkeit der Suchttherapie eine weitere effektive pharmakologische Rückfallprophylaxe zur Verfügung.

Mit der aktiven Immunisierung hat die Behandlung der Kokainabhängigkeit ein spannendes Neuland betreten. Sollte sie sich in den laufenden Erprobungen am Menschen als erfolgreich erweisen, so wäre die Tür zu einem neuen Therapieansatz mit eventuell großem Entwicklungspotenzial geöffnet. Noch bleibt dies begründete Hoffnung.

\section{Korrespondierender Autor \\ Prof. Dr. G. Wiesbeck \\ Bereich Abhängigkeitserkrankungen, Universitäre Psychiatrische Kliniken Wilhelm-Klein-Strasse 27, 4025 Basel gerhard.wiesbeck@upkbs.ch}

Interessenkonflikt. Es besteht kein Interessenkonflikt. Der korrespondierende Autor versichert, dass keine Verbindungen mit einer Firma, deren Produkt in dem Artikel genannt ist, oder einer Firma, die ein Konkurrenzprodukt vertreibt, bestehen. Die Präsentation des Themas ist unabhängig und die Darstellung der Inhalte produktneutral.

\section{Literatur}

1. Baird TJ, Deng SX, Landry DW (2000) Natural and artificial enzymes against cocaine. I: monoclonal antibody $15 \mathrm{~A} 10$ and the reinforcing effects of cocaine in rats. J Pharmacol Exp Ther 295: 1127-1134

2. Bartzokis G, Beckson M, Newton T et al. (1999) Selegiline effects on cocaine-induced changes in medial temporal lobe metabolism and subjective ratings of euphoria. Neuropsychopharmacology 20 : 582-590

3. Basmadjian GP, Singh S, Sastrodjojo B (1995) Generation of polyclonal catalytic antibodies against cocaine using transition state analogs of cocaine conjugated to diphtheria toxoid. Chem Pharm Bull (Tokyo) 43: 1902-1911

4. Bonese KF, Wainer BH, Fitch FW et al. (1974) Changes in heroin self-administration by a rhesus monkey after morphine immunisation. Nature 252: 708-710

5. Brodie JD, Figueros E, Dewey SL (2003) Treating cocaine addiction: from preclinical to clinical trial experience with gamma-vinyl GABA. Synapse 50: 261-265

6. Caroldi S, DeParis P (1995) Comparative effects of two dithiocarbamates disulfiram and thiram on adrenal catecholamine content and on plasma dopamine $\beta$-hydroxylase activity. Arch Toxicol 69 690-693

7. Carroll K, Ziedonis D, O'Malley S et al. (1993) Pharmacologic interventions for alcohol- and cocaineabusing individuals. A pilot study of disulfiram and naltrexone. Am J Addict 2: 77-79

8. Carroll KM, Fenton LR, Ball SA et al. (2004) Efficacy of disulfiram and cognitive behaviour therapy in cocain-dependent outpatients. Arch Gen Psychiatry 61: 264-272
9. Carroll KM, Nich C, Ball SA et al. (1998) Treatment of cocaine and alcohol dependence with psychotherapy and disulfiram. Addiction 93: 713-728

10. Cashman JR, Berkman CE, Underiner GE (2000) Catalytic antibodies that hydrolyze (-)-cocaine obtained by a high-throughput procedure. J Pharmacol Exp Ther 293: 952-961

11. Dackis CA, Lynch KG, Yu E et al. (2003) Modafinil and cocaine: a double-blind, placebo-controlled drug interaction study. Drug Alcohol Depend 70: 29-37

12. Dackis CA, Kampman KM, Lynch KG et al. (2005) A double-blind, placebo-controlled trial of modafinil for cocaine dependence. Neuropsychopharmacology 30: 205-211

13. Drogen- und Suchtbericht (2005) Drogen- und Suchtbericht 2005. http://www.bmgs.bund.de/ download/broschueren/A601-05.pdf

14. Europäische Beobachtungsstelle für Drogen und Drogensucht (2003) Jahresbericht 2003: Stand der Drogenproblematik in der Europäischen Union und in Norwegen. http://anualreport.emcdda.eu.int. Amt für amtliche Veröffentlichungen der Europäischen Gemeinschaften, Luxemburg

15. Fadda P, Scherma M, Fresu A et al. (2003) Baclofen antagonizes nicotine-, cocaine-, and morphine-induced dopamine release in the nucleus accumbens of rat. Synapse 50: 1-6

16. Foltin RW, Fischman MW (1998) Effects of "binge" use of intravenous cocaine in methadone-maintained individuals. Addiction 93: 825-836

17. Fox BS, Kantak KM, Edwards MA et al. (1996) Efficacy of a therapeutic cocaine vaccine in rodent models. Nat Med 2: 1129-1132

18. Gawin FH, Riordan C, Kleber H (1985) Methylphe nidate treatment of cocaine abusers without attention deficit disorder: a negative report. Am J Drug Alcohol Abuse 11: 193-197

19. George TP, Chawarski MC, Pakes J (2000) Disulfiram versus placebo for cocaine dependence in buprenorphine-maintained subjects: a preliminary trial. Biol Psychiatry 47: 1080-1086

20. Goldstein M, Anagoste B, Lauber E (1964) Inhibition of dopamine $\beta$-hydroxylase by disulfiram. Life Sci 3: 763-767

21. Grabowski J, Roache J, Schmitz J et al. (1997) Replacement medication for cocaine dependence: methylphenidate. J Clin Psychopharmacol 17: 485-488

22. Gonzales G, Sevarine K, Sofuoglu M et al. (2003) Tiagabine increases cocaine-free urines in cocainedependent methadone-treated patients: results of a randomized pilot study. Addiction 98: 16251632

23. Hadorn DC, Baker D, Hodges JS et al. (1996) Rating the quality of evidence for clinical practice guideline. J Clin Epidemiol 49: 749-754

24. Hall FS, Sora I, Drgonova J et al. (2004) Molecular mechanisms underlying the rewarding effects of cocaine. Ann N Y Acad Sci 1025: 47-56

25. Hameedi FA, Rosen MI, McCance-Katz EF et al. (1995) Behavioral, physiologic and pharmacologic ineraction of cocaine and disulfiram in humans. Biol Psychiatry 37: 560-563

26. Hearn WL, Flynn DD, Hime GW et al. (1991) Cocaethylene: a unique cocaine metabolite displays high affinity for the dopamine transporter. J Neurochem 56: 698-701

27. Higgins ST, Budney AJ, Bickel WK et al. (1993) Disulfiram therapy in patients abusing cocaine and alcohol. Am J Psychiatry 150: 675-676

28. Honjo T, Netter KJ (1969) Inhibition of drug demethylation by disulfiram in vivo and in vitrol. Biochem Pharmacol 18: 2681-2683
29. Houtsmuller EJ, Notes LD, Newton T et al. (2004) Transdermal selegiline and intravenous cocaine: safety and interactions. Psychopharmacology (Berl) 172: 31-40

30. Izenwasser $\mathrm{S}$ (2004) The role of the dopamine transporter in cocaine abuse. Neurotox Res 6: 379-383

31. Kampman KM, Pettinati H, Lynch KG et al. (2004) A pilot trial of topiramate for the treatment of cocaine dependence. Drug Alcohol Depend 75: 233-240

32. Kantak KN, Collins SL, Lipman EG et al. (2000) Evaluation of anti-cocaine antibodies and a cocaine vaccine in a rat self-administration mode. Psychopharmacology (Berl) 148: 251-262

33. Kosten TR, Rosen M, Bond J et al. (2002) Human therapeutic cocaine vaccine: safety and immunogenicity. Vaccine 20: 1196-1204

34. Kuwahata T, Ishimatsu M, Kidani Y et al. (2002) Effects of methylphenidate on the inhibitory postsynaptic potential in rat locus coeruleus neurons. Kurume Med J 49: 185-190

35. Landry DW, Zhao K, Yang GX et al. (1993) Antibody-catalysed degradation of cocaine. Science 259 1899-1901

36. Leri F, Bruneau J, Stewart J (2003) Understanding polydrug use: review of heroin and cocaine co-use. Addiction 98: 7-22

37. Lile JA, Stoops WW, Allen TS et al. (2004) Baclofen does not alter the reinforcing, subject-rated or cardiovascular effects of intranasal cocaine in humans. Psychopharmacology (Berl) 171: 441-449

38. Lima MS, Reisser AAP, Soares BGO et al. (2002) Pharmacological treatment of cocaine dependence: a systematic review. Addiction 97: 931-949

39. Ling W, Shoptaw S, Majewska D (1998) Baclofen as a cocaine anti-craving medication: a preliminary clinical study. Neuropsychopharmacology 18 : 403-404

40. Maffli E, Kuntsche S, Delgrande JM et al. (2003) Ambulante Suchtberatung 2001-Statistik der ambulanten Behandlung und Betreuung im Alkoholund Drogenbereich. Bundesamt für Statistik (BFS) Neuchatel

41. McCance-Katz EF, Kosten TR, Jatlow P (1998) Chronic disulfiram treatment effects on intranasal cocaine administration-initial results. Biol Psychiatry 43: 540-543

42. McCance-Katz EF, Kosten TR, Jatlow P (1998) Concurrent use of cocaine and alcohol is mor potent and potentially more toxic than use of either alone - a multiple-dose study. Biol Psychiatry 44: 250-259

43. McCance-Katz EF, Kosten TR, Jatlow P (1998) Disulfiram effects on acute cocaine administration. Drug Alcohol Depend 52: 27-39

44. Morell V (1993) Enzyme may blunt cocaine's action. Science 259: 1828

45. Narring F, Tschumper A, Inderwildi Bonivento $L$ (2002) Gesundheit und Lebensstil 16- bis 20 Jähriger in der Schweiz. SMASH 2002: Swiss multicenter adolescent study on health 2002. Institut universitaire de médecine sociale et préventive, Lausanne

46. Newton T, Kalechstein A, Beckson M et al. (1999) Effects of selegiline pre-treatment on response to experimental cocaine administration. Psychiatry Res 87: 101-106

47. Nich C, McCance-Katz EF, Petrakis IL et al. (2004) Sex differences in cocaine-dependent individuals' response to disulfiram treatment. Addict Behav 29: 1123-1128

48. NIDA (2005) Topiramate shows promise in cocaine addiction. NIDA Notes 19: 1-6

49. Parran TV, Jasinski DR (1991) Intravenous methylphenidate abuse: prototype for prescription drug abuse. Arch Intern Med 151: 781-783 
50. Ritz MC, Lamb RJ, Goldberg SR et al. (1987) Cocaine receptors on dopamine transporters are related to self-administration of cocaine. Science 237: $1219-1223$

51. Roache J, Grabowski J, Schmitz J et al. (2000) Laboratory measures of methylphenidate effects in cocaine-dependent patients receiving treatment. J Clin Psychopharmacol 20: 61-68

52. Rocha BA, Fumagalli F, Gainetdinov RR et al. (1998) Cocaine self-administration in dopamine-transporter knockout mice. Nat Neurosci 1: 132-137

53. Scheid R, Schindler E, Biniek R (1999) Kokaininduzierte akute ZNS-Erkrankungen. Nervenarzt 70: 315-321

54. Schiffer WK, Marsteller D, Dewey SL (2003) Sub-chronic gamma-vinyl GABA (vigabatrin) inhibits cocaine-induced increases in nucleus accumbens dopamine. Psychopharmacology (Berl) 168: 339-343

55. Shaffer HJ, Eber GB (2002) Temporal progression of cocaine dependence symptoms in the US National Comorbidity Survey. Addiction 97: 543-554

56. Shoptaw S, Yank X, Rotheram-Fuller EJ et al. (2003) Randomized placebo-controlled trial of baclofen for cocaine dependence: preliminary effects for individuals with chronic patterns of cocaine use. J Clin Psychiatry 64: 1440-1448

57. Somoza EC, Winhusen TM, Rotrosen JP et al. (2004) An open-label pilot study of methylphenidate in the treatment of cocaine dependent patients with adult attention deficit/hyperactivity disorder. J Addict Dis 23: 77-92

58. Volkow ND, Ding YS, Fowler JS et al. (1995) Is methylphenidate like cocaine? Studies on their pharmacokinetics and distribution in the human brain. Arch Gen Psychiatry 52: 456-463

59. Volkow ND, Wang GJ, Fowler JS et al. (1999) Blockade of striatal dopamine transporters by intravenous methylphenidate is not sufficient to induce self-reports of "high". J Pharmacol Exp Ther 288: 14-20

60. Volkow ND, Wang GJ, Fowler JS et al. (1998) Dopamine transporter occupancies in the human brain induced by therapeutic doses of oral methylphenidate. Am J Psychiatry 155: 1325-1331

61. Yang G, Chun J, Arakawa-Uramoto H (1996) Anticocaine catalytic antibodies: a synthetic approach to improved antibody diversity. J Am Chem Soc 118: $5881-5890$

Nervenarzt 2006 · 77:1064-1070 DOI 10.1007/s00115-006-2165-0

Online publiziert: 6. September 2006 C Springer Medizin Verlag 2006

H. B. Huttner · E. Jüttler · A. Hug · M. Köhrmann · P. D. Schellinger · T. Steiner Abteilung für Neurologie, Ruprecht-Karls-Universität Heidelberg

\section{Antikoagulanzienasso- ziierte Hirnblutungen}

\section{Nervenarzt (2006) 77:671-681}

In dem Beitrag „Antikoagulanzienassoziierte Hirnblutungen“ von Huttner et al. wurde, bei korrekter Nummerierung der Seitenzahlen, der Text auf den Seiten 675 und 676 vertauscht. Wir bitten Sie, diesen technischen Fehler zu entschuldigen.
Die Online-Version des Originalartikels können Sie unter DerNervenarzt.de finden. 